\title{
Predicting change in quality of life from age 79 to 90 in the Lothian Birth Cohort 1921
}

\author{
Caroline E. Brett ${ }^{1,2} \cdot$ Dominika Dykiert ${ }^{2,3} \cdot$ John M. Starr ${ }^{2,4} \cdot$ lan J. Deary ${ }^{2,5}$
}

Accepted: 15 November 2018 / Published online: 23 November 2018

(c) The Author(s) 2018

\begin{abstract}
Purpose Quality of life (QoL) decreases in very old age, and is strongly related to health outcomes and mortality. Understanding the predictors of QoL and change in QoL amongst the oldest old may suggest potential targets for intervention. This study investigated change in QoL from age 79 to 90 years in a group of older adults in Scotland, and identified potential predictors of that change.

Method Participants were members of the Lothian Birth Cohort 1921 who attended clinic visits at age $79(n=554)$ and 90 $(n=129)$. Measures at both time points included QoL (WHOQOL-BREF: four domains and two single items), anxiety and depression, objective health, functional ability, self-rated health, loneliness, and personality.

Results Mean QoL declined from age 79 to 90 . Participants returning at 90 had scored significantly higher at 79 on most QoL measures, and exhibited better objective health and functional ability, and lower anxiety and depression than non-returners. Hierarchical multiple regression models accounted for $20.3-56.3 \%$ of the variance in QoL at age 90 . Baseline QoL was the strongest predictor of domain scores (20.3-35.6\% variance explained), suggesting that individual differences in QoL judgements remain largely stable. Additional predictors varied by the QoL domain and included self-rated health, loneliness, and functional and mood decline between age 79 and 90 years.

Conclusions This study has identified potential targets for interventions to improve QoL in the oldest old. Further research should address causal pathways between QoL and functional and mood decline, perceived health and loneliness.
\end{abstract}

Keywords Quality of life $\cdot$ Functional status $\cdot$ Health $\cdot$ Older adults $\cdot$ Ageing $\cdot$ Mood $\cdot$ Functional decline

Electronic supplementary material The online version of this article (https://doi.org/10.1007/s11136-018-2056-4) contains supplementary material, which is available to authorized users.

Caroline E. Brett

c.e.brett@ljmu.ac.uk

1 Natural Sciences and Psychology, Liverpool John Moores University, Byrom Street, Liverpool L3 3AF, England, UK

2 Centre for Cognitive Ageing and Cognitive Epidemiology, University of Edinburgh, Edinburgh, UK

3 Anna Freud National Centre for Children and Families and University College London, London, UK

4 Alzheimer Scotland Dementia Research Centre, University of Edinburgh, Edinburgh, UK

5 Department of Psychology, University of Edinburgh, Edinburgh, UK

\section{Introduction}

Older age brings increasing vulnerability as a result of physical and functional decline, and concomitant burdens on health and social care services. Maintaining good health and wellbeing are often portrayed as markers of healthy or successful ageing [1-4] and are a target for policymakers and health professionals alike [4].

Defined as "individuals' perceptions of their position in life in the context of the culture and value systems in which they live and in relation to their goals, expectations, standards and concerns" [5], quality of life (QoL) has been characterised as a global aggregated measure, incorporating both objective and subjective indicators. As such, it is thought to be more influenced by situational factors such as material or social circumstances than measures of subjective wellbeing, such as life satisfaction or positive affect, which are more strongly associated with psychological factors [6-8]. Poor QoL has been shown to predict a range of negative health 
outcomes, including mortality [9-13]. Consequently, QoL is increasingly regarded as an important outcome measure for interventions aimed at improving health outcomes or reducing health inequalities [14].

The relationship between age and subjective ratings of wellbeing and QoL is complex $[15,16]$. Research generally suggests a U-shaped relationship across the life course [17-20], with a decline in QoL and wellbeing amongst the oldest old aged 75 years and over $[18,21,22]$. This relationship appears to differ depending on the measure used. Growing evidence suggests that, when measured in terms of life satisfaction, wellbeing is relatively stable even amongst the oldest old and 'bounces back' following negative life events (including spousal death) to a set point [23-25], which itself is determined largely by psychological factors developed over the life course $[26,27]$. In contrast, QoL appears to fluctuate over time, with individual trajectories determined predominantly by changing circumstances rather than age $[18,28,29]$.

Understanding the predictors of individual differences in both the current level of and change in QoL over time amongst older adults is important for identifying potential targets for intervention. Although the majority of research to date has been cross-sectional, it has identified a number of strong candidates for potential predictors of QoL in old age, including functional status (activities of daily living (ADLs)) [18, 30-32], health (predominantly chronic conditions) [30, 31, 33], depression [20, 30, 33-37], anxiety [14, $30]$, marital status [18, 28, 31], quality of social contacts $[30,38]$, socioeconomic conditions [31, 38], and personality $[30,37]$. Longitudinal studies have suggested that current circumstances influence QoL judgements more than early life circumstances [39], which might exert influence on QoL indirectly through current circumstances such as property ownership and health [40]. Using data from the Berlin Aging Study, Baltes and colleagues identified a psychological profile amongst their oldest participants with the highest wellbeing which included many of the above factors [41].

Studies investigating predictors of change in QoL in older age are scarce, especially amongst the oldest old. Webb et al. [28] showed that QoL decreased over 4 years amongst over$50 \mathrm{~s}$, that decline was associated with increased depression and difficulties with ADLs, but that improvements in family relationships, neighbourhood, and perceived financial position counteracted decline in QoL. Chan et al. [33] found that increased physical illness, depressive symptoms, and difficulties with instrumental ADLs, were all associated with decreased QoL over 12 months in over-65s diagnosed with a depressive disorder. Some researchers suggest that, over time, older adults place increasing emphasis on health and mobility when determining their QoL [30, 39]. With growing numbers of people reaching their ninth decade and experiencing declining physical and cognitive capabilities, investigation of how QoL changes during this time is timely and essential [42].

The current study aims to investigate change in QoL between the ages of 79 and 90 years in a group of older adults in Scotland, and to identify potential predictors of that change. We have previously shown that depression, emotional stability, conscientiousness, social class, living alone, and health had significant cross-sectional associations with QoL in this Lothian Birth Cohort 1921 (LBC1921) group at age 79, and that determinants differed between QoL domains [37]. In the present study, we hypothesised that mean QoL would decline from age 79 to age 90 and that baseline QoL would be the strongest predictor of current QoL. Based on our own and previous research, we also hypothesised that increases (age 79-90) in depression, changes in living-alone status, health, and functional status, personality, and occupational social class would all be associated with changes in QoL.

\section{Methods}

\section{Participants}

The participants were all members of the LBC1921, which has been described in detail elsewhere [43]. The LBC1921 Study consists of 550 individuals (238 men), most of whom participated in the Scottish Mental Survey 1932 aged around 11 years [44]. Between 1999 and 2001, participants-all of whom were living independently and aged around 79 years - undertook detailed cognitive and physical testing, and answered questions related to their health, occupation, and lifestyle. At age 80-81, 497 participants completed a questionnaire which included QoL items, and, at age 81, 467 participants completed a personality questionnaire. Additional waves of follow-up testing were completed at age 83 and 87 [43].

In 2011, at age 90 years, all participants, except those who had died $(n=190,34.5 \%)$, withdrawn $(n=73,13.3 \%)$, lost contact $(n=15,2.7 \%)$, were not well enough, or were ineligible to participate $(n=105,19.1 \%$; this included participants with a diagnosis of dementia), or were unable to take part for other reasons ( $n=27,4.9 \%$; moved away or had caring responsibilities), were invited to a fourth wave of follow-up testing [45]. Participants with dementia were excluded due to the study's emphasis on non-pathological cognitive ageing; sensitivity analyses have shown that incipient dementia had little influence on key findings from this cohort [46]. 129 participants attended a clinic visit involving comprehensive cognitive and physical testing and a structured interview, as per wave 1 . They also completed an extensive questionnaire, which included a repeat of the QoL items. The current study uses data from wave 1 and wave 
4 clinic visits, and the QoL and personality questionnaires completed at age $80 / 81$.

\section{Measures}

\section{Repeated measures}

Quality of life QoL was measured at waves 1 and 4 (ages 79 and 90) using the WHOQOL-BREF, which is a wellvalidated abbreviated version of the WHOQOL-100 quality of life assessment containing 26 items [47-50]. The WHOQOL-BREF contains one item from each of the 24 facets of QoL included in the WHOQOL-100 and which produce scores on four QoL domains: physical (7 items, e.g. "How satisfied are you with your sleep?"), psychological (8 items, e.g. "To what extent do you feel your life to be meaningful?"), social (3 items, e.g. "How satisfied are you with your personal relationships?"), and environment (9 items, e.g. "How satisfied are you with the conditions of your living place?"), and two additional items measuring overall QoL and general health. Participants are asked to consider the extent to which each item reflects their experiences over the last 2 weeks, and indicate their response on a 5-point Likert-type scale. As per the WHOQOL-BREF manual [48], domain scores were calculated by multiplying the mean score across all items relating to that domain by 4 , resulting in a score out of 20 . The single items were scored out of 5. In all cases, higher scores indicated better QoL. For the purposes of correlational and ordinal regression analysis, the single-item scores were collapsed into either three (poor/neither poor nor good, good, very good) or four (very/ dissatisfied, neither, satisfied, very satisfied) categories for the QoL and health QoL items, respectively.

As reported elsewhere [37], one question (q21: "How satisfied are you with your sex life?") was judged to be inappropriate for this age group and reworded as "How satisfied are you with the support you get from your family?" Missing values were handled using a pro-rating technique, whereby single missing values in each domain were replaced with the mean of the remaining items from that domain. Where multiple values were missing in a domain, these were not replaced. Cronbach's alphas indicated acceptable to good internal consistency for all domains at both waves (wave 1: $\alpha=0.730$ to 0.844 ; wave $4: \alpha=0.617$ to 0.751 ).

Scores on the four domains and two single items at wave 1 were subtracted from their wave 4 counterparts in order to calculate raw change in QoL over time, with positive values indicating increased QoL.

Current mood The Hospital Anxiety and Depression Scale (HADS) [48] was used to measure symptoms of anxiety and depression at waves 1 and 4. The HADS consists of 7 items each for anxiety and depression, each scored on a 3-point scale giving a maximum score of 21 . Scores of $8+$ are seen to suggest possible cases of anxiety disorders or depression [51, 52].

Functional ability The Townsend Functional Ability Scale [53] was used to measure current functional status at waves 1 and 4. Participants were asked how able they were to complete a series of nine everyday tasks. Each was scored on a 3 -point scale with a maximum score of 18 ; higher scores indicated greater impairment.

Objective health status Biomarkers are considered key indicators of healthy ageing $[54,55]$, and are more reliable than self-report measures of health or health behaviours [56].

Grip strength, peak expiratory flow (PEF), and Body Mass Index (BMI) were used as objective measures of health status. All were measured during the clinic visit at waves 1 and 4 . Grip strength has been shown to be related to future health outcomes [57] and cognition [58] amongst older adults. This was measured using a dynamometer; three measurements were taken for each hand and the best of all six used in the analyses. PEF has been shown to be a valid index of health status in older adults and independently associated with ADLs, hospitalisation, and subjective mortality risk assessment [59, 60]. This was measured using a spirometer. Participants were asked to blow into the apparatus for as long as they could; the best of three measurements was taken. Both variables were adjusted for sex and height at the time of testing and the standardised residuals used in subsequent analyses. High and low BMI has been associated with lower health-related QoL and physical functioning in older adults [61]. Height and weight were measured during each clinic visit.

Residualised change scores for the mood, functional ability, and objective health measures were calculated by regressing equivalent wave 4 scores on wave 1 (baseline) scores. In accordance with the directionality of the original measures, higher change scores indicate worsening anxiety, depression, and functional ability, and less decline in (or relatively improving) grip strength and lung function.

Living-alone change Participants were asked at both waves whether they lived alone or not. This was converted into a change score based on the anticipated impact of the change from wave 1 to wave 4 , as follows: $-1=$ change from not alone to alone $(n=32), 0=$ no change $(n=90), 1=$ change from alone to not alone $(n=6)$.

\section{Baseline (wave 1; age 79) measures}

Social class Participants were asked at wave 1 to provide details of their highest status occupation. Using the 1951 
Classification of Occupations [62], this was used to derive their occupational social class within five groupings: I (professional), II (managerial and technical), III (skilled), IV (semi-skilled), and V (unskilled). Female participants were also asked for their husband's occupation (where applicable) and the higher of the two was used to represent their social class. Social class was deemed to have both a distal and proximal influence on QoL through its effect on income and therefore material circumstances in old age.

Personality The 50-item version of the International Personality Item Pool (IPIP) [63-65] was used at wave 1 to measure scores on the Big Five personality traits of extraversion, agreeableness, conscientiousness, emotional stability, and intellect/imagination. Scores for each trait were calculated by summing responses to 10 items placed on a 5 -point Likert-type scale. For the present analysis, only those traits that previous research has shown to be strongly associated with QoL $[37,66]$ were included, i.e. conscientiousness and emotional stability (the inverse of neuroticism).

Loneliness Loneliness was measured at wave 1 using a single item, "At the present moment do you feel lonely?", answered from a choice of five options (scored 5-1, higher scores indicating higher loneliness): most of the time, quite often, only occasionally, seldom, and never.

\section{Current (wave 4; age 90) measures}

Self-rated health Self-rated health was measured at wave 4 using a single item: "How would you rate your health just now?", answered from five options (scored 5-1): excellent, very good, good, fair, and poor.

\section{Statistical analysis}

All statistical analyses were carried out using SPSS Statistics for Windows, Version 24.0 (IBM Corp, Armonk NY).

Independent sample $t$ tests were conducted to compare participants who returned for wave 4 with those who did not on all baseline measures. Paired $t$ tests were then conducted to identify significant differences between all repeated measures from baseline and wave 4 .

As all variables were found to be non-normally distributed, Spearman's rho bivariate correlations were calculated between all baseline and change variables, and the QoL measures at both waves. To correct for multiple testing, the false discovery rate (FDR) was controlled $(\alpha=0.05)$ using a procedure described in Benyamini and Hochberg [67]. To enhance model parsimony, only variables with correlations that were significantly associated with the relevant $\mathrm{QoL}$ measure below the critical $p$ value identified by the FDR calculation were included in subsequent regression analyses.
A series of hierarchical multiple linear regression analyses were carried out with each of the four QoL domains as the outcome variable. In order to test the additional contribution made by each category of predictor variable, baseline scores on the QoL measure were included at the first step, baseline predictors (including personality) at the second step, change variables (functional ability and mood) at the third step, and current measures at the fourth step. Finally, proportional odds logit ordinal regression was carried out with the two single QoL items as the outcome variables and variables at each level (baseline QoL, other baseline, change, current) added in turn. Again, FDR calculations were carried out for each analysis type to control for multiple testing.

\section{Results}

Descriptive statistics for the QoL and predictor variables at both waves of testing are shown in Table 1. For wave 1, these are shown for all participants and by returners versus nonreturners. Participants who did not return for wave 4 scored significantly lower on physical, psychological, and environmental QoL, and on the two single QoL items at wave 1, as well as reporting significantly higher anxiety, depression, and functional limitations, scoring significantly lower on emotional stability, and exhibiting lower grip strength and lung function (these differences were only statistically significant in women and men, respectively).

Participants who completed both waves scored significantly lower at age 90 than they had done at age 79 on all QoL measures except the social domain (see Table 1; Fig. 1). They also reported significantly more functional limitations, lower anxiety, and higher depression, and exhibited significantly lower grip strength and lung function at age 90 than they had done at age 79 .

Table 2 contains the results of Spearman's bivariate correlations between the six QoL measures from wave 4 and all the predictor variables, including baseline QoL. Most of the strongest associations were between the comparable QoL measures at baseline (wave 1) and wave 4, with rho values ranging from 0.36 (QoL item) to 0.61 (psychological QoL). Higher physical QoL and the single health QoL item were both associated with lower age-79-90 reduction in and higher current functional ability, lower age-79-90 increase in and lower current depression, and higher current selfrated health. Higher physical QoL was also associated with higher current grip strength. Higher psychological QoL was associated with higher baseline emotional stability, lower baseline loneliness, lower age-79-90 increase in and lower current anxiety and depression, and higher current self-rated health. Social QoL was not significantly associated with any predictor variables. Higher environmental QoL was associated with higher emotional stability and occupational social 
Table 1 Means, etc. for WHOQOL-BREF domains and items, and all predictor variables for both waves

\begin{tabular}{|c|c|c|c|c|c|c|c|}
\hline & All w1 & & W1 non-returned & $\mathrm{W} 1$ returned $^{\mathrm{a}}$ & W4 & & Change (w1-w4) \\
\hline & Mean (SD) & $\alpha$ & Mean (SD) & Mean (SD) & Mean (SD) & $\alpha$ & Mean (SD) \\
\hline$N$ & $460-554$ & & $325-425$ & 129 & $124-129$ & & $123-129$ \\
\hline Physical QoL & $14.86(2.78)$ & 0.844 & $14.64(2.84)$ & $15.62 * *(2.37)$ & $13.78(2.51)$ & 0.751 & $-1.89 * * *(2.23)$ \\
\hline Psychological QoL & $15.28(2.04)$ & 0.764 & $15.12(2.18)$ & $15.74 * *(1.55)$ & $14.49(2.00)$ & 0.748 & $-1.24 * * *(1.63)$ \\
\hline Social QoL & $17.28(2.36)$ & 0.730 & $17.15(2.46)$ & $17.61(2.00)$ & $17.51(2.30)$ & 0.739 & $-0.07(2.26)$ \\
\hline Environment QoL & $16.48(2.10)$ & 0.785 & $16.27(2.18)$ & $17.06 * * *(1.67)$ & 15.94 (1.64) & 0.617 & $-1.09 * * *(1.65)$ \\
\hline QoL item & $4.28(0.71)$ & - & $4.21(0.73)$ & $4.46^{* * * *}(0.60)$ & $3.98(0.70)$ & - & $-0.49 * * *(0.74)$ \\
\hline QoL health item & $3.67(0.93)$ & - & $3.58(0.94)$ & $3.98 * * *(0.81)$ & $3.44(0.96)$ & - & $-0.56 * * *(0.91)$ \\
\hline Townsend & $2.26(2.80)$ & 0.783 & $2.51(2.98)$ & $1.44 * * *(1.87)$ & $5.10(4.56)$ & \pm & $3.66 * * *(3.98)$ \\
\hline Grip strength: men & $26.74(8.97)$ & - & $26.40(9.03)$ & $27.93(8.75)$ & $20.71(8.18)$ & - & $-7.30 * * *(5.13)$ \\
\hline Grip strength: women & $26.39(9.23)$ & - & $25.48(8.89)$ & $29.19 * *(9.72)$ & $21.10(8.43)$ & - & $-8.15^{* * *}(4.73)$ \\
\hline PEF: men & $277.84(140.00)$ & - & $265.90(136.92)$ & $320.10 *(143.98)$ & $243.84(121.71)$ & - & $-78.82 * * *(104.89)$ \\
\hline PEF: women & $267.80(126.96)$ & - & $260.32(126.05)$ & 290.69 (127.79) & $220.32(97.15)$ & - & $-70.63 * * *(91.24)$ \\
\hline Anxiety & $5.20(3.31)$ & 0.758 & $5.42(3.43)$ & $4.45 * *(2.72)$ & $3.71(2.87)$ & 0.762 & $-0.69 * *(2.34)$ \\
\hline Depression & $3.53(2.33)$ & 0.614 & $3.69(2.40)$ & $3.01 * *(1.97)$ & $3.82(2.35)$ & 0.521 & $0.86 * * *(2.32)$ \\
\hline Living alone (\%) & $48.0 \%$ & - & $48.3 \%$ & $46.9 \%$ & $66.7 \%$ & - & \\
\hline IPIP C & $38.71(6.08)$ & 0.766 & 38.63 (6.09) & $38.96(6.05)$ & - & - & - \\
\hline IPIP ES & $34.24(8.13)$ & 0.872 & $33.53(8.20)$ & $36.37 * *(7.59)$ & - & - & - \\
\hline Loneliness & $1.97(1.02)$ & - & $2.01(1.05)$ & $1.83(0.93)$ & - & - & - \\
\hline Social class & $2.24(0.88)$ & - & $2.30(0.87)$ & $2.04(0.89)$ & & - & \\
\hline Self-rated health & - & - & - & - & $3.56(0.87)$ & - & - \\
\hline
\end{tabular}

For W1 returned column, $\mathrm{p}$ values reflect differences between returners and non-returners. For change column, $\mathrm{p}$ values reflect differences between wave 1 and wave $4 . \pm$ Item-level data were not available for the Townsend Functional Ability Scale at wave 4

$* t$ Test $p<.05 ; * * t$ test $p<.01 ; * * * t$ test $p<.001$

${ }^{\mathrm{a}}$ These are individuals who attended both wave 1 and returned for wave 4
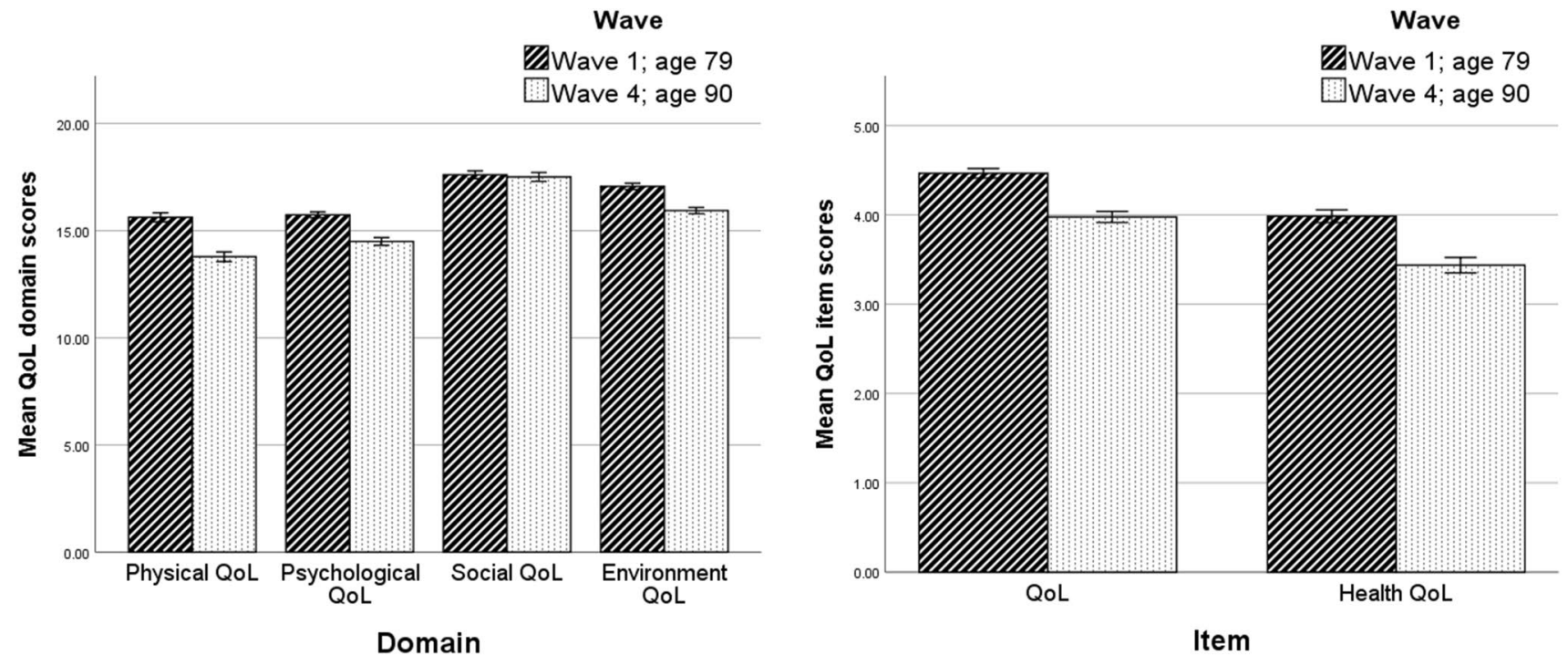

Fig. 1 Mean scores on QoL domains and single QoL items for returners at age 79 and 90 (error bars represent standard errors)

class, lower baseline loneliness, lower current anxiety and depression, and higher current self-rated health. The single QoL item was associated with lower baseline loneliness, lower age-79-90 reduction in and higher current functional ability, lower age-79-90 increase in and current depression, and higher current self-rated health and lung function. 


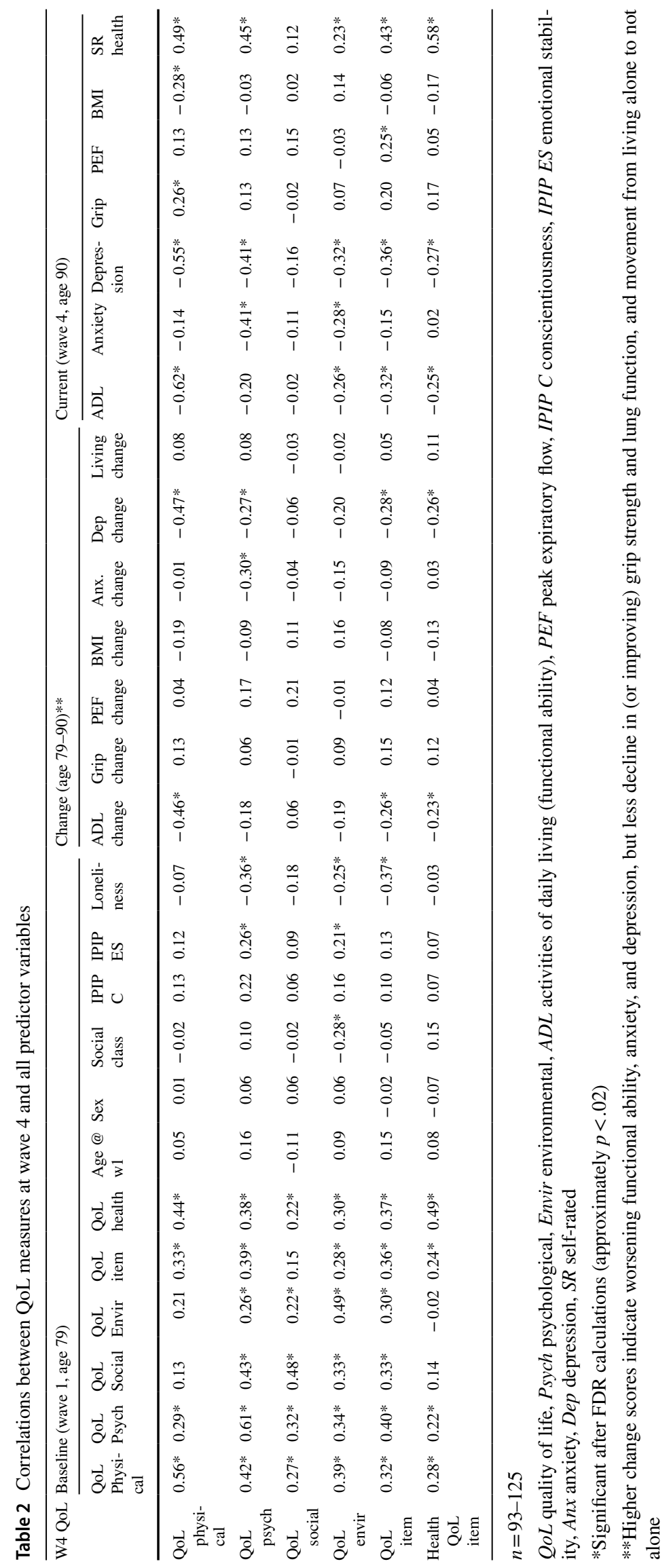


The results of the final models from the hierarchical regression analyses for each QoL domain are in Table 3. The models account for between $20.3 \%$ (social QoL) and $56.3 \%$ (physical QoL) of the variance in the QoL measures. For all the domain scores, the strongest predictor was baseline QoL in the given domain, accounting for the majority of the variance explained. Physical QoL was also significantly predicted by age-79-90 change in functional ability and depression. Psychological QoL was significantly predicted by age-79-90 change in anxiety, and current selfrated health. Social QoL and environmental QoL were only significantly predicted by the baseline scores. Supplementary Tables S1-S4 show the results for each step of the hierarchical linear regression analyses. Overall, baseline QoL explained between 20.3 and $35.6 \%$ of the variance in the equivalent current QoL measures, other baseline predictors contributed between 0.4 and $3.1 \%$ additional variance, age 79-90 change variables contributed between 8.3 and $23.3 \%$, and current predictors contributed between 2.3 and $9.2 \%$.

The results of the ordinal regression analyses are shown in Table 4 and Supplementary Tables S5, S6. In the final models, the QoL item was significantly predicted by baseline loneliness and current self-rated health. The health QoL item was significantly predicted by baseline health QoL and current self-rated health. Pearson's goodness-of-fit test indicated that for both models the observed probabilities did not deviate from the probabilities predicted by the multinomial regressions, suggesting that the models fit the data well $\left(\chi^{2}=183.36, p=.356\right.$ and $\left.\chi^{2}=370.08, p=.293\right)$. For the QoL item, baseline QoL significantly predicted current QoL for the first two models, but this effect was attenuated when current predictors were added.

\section{Discussion}

This study is one of the first to investigate predictors of change in QoL across 11 years of old-old age. The results show that QoL declined significantly across most domains and the QoL and health single items between 79 and 90, as did functional ability and objective health status. This is in keeping with the results of previous studies [18, 22, 28, 39]. Baseline QoL, functional ability, grip strength, lung function, and emotional stability were all higher and anxiety and depression lower in returners compared to non-returners, pursuant to research suggesting that retention in longitudinal studies is higher amongst those who are advantaged [28]. Given the principal reason for attrition in the LBC1921 was mortality, poor health, or poor function [45, 68], this finding further supports the suggestion that poor QoL is associated with poorer health outcomes and mortality $[9,11-13,69]$.

The findings suggest that, for the four QoL domains, QoL at age 90 is most strongly associated with QoL earlier in old age. Lower physical QoL was also associated with worsening depression and functional ability and psychological QoL with increasing anxiety. These results support previous research $[18,30]$. The single QoL item was associated with baseline loneliness and current self-rated health, while the health QoL item was associated with baseline health QoL and current self-rated health.

The largest single determinant of QoL domain scores at age 90 was QoL at age 79. This supports previous research suggesting that QoL is comparatively stable within individuals in old age and 'bounces back' from adversity [29, 70]. Much research into healthy ageing has focussed on individual differences in psychological resources such as resilience, optimism, personality, coping, sense of coherence, and perceived social support. These are thought to influence individuals' ability to cope and maintain their wellbeing in the face of adversity, and, crucially, their subjective experience and ratings of their health and wellbeing [41, 70]. However, QoL stability contrasts with research suggesting QoL is sensitive to changes in physical health [6]. In the present study, the final models only explained at most a third of the variance in social QoL, and environmental QoL. This suggests that factors not included in this analysis are likely to be important and more research is needed to identify them. Of note, social QoL-measured here as satisfaction with personal relationships, friends, and family - was not explained by any other factors, suggesting it is unaffected by physical and health limitations and may be a suitable target for intervention amongst the oldest old. This complements current approaches towards improving wellbeing in later life $[42,71]$.

In contrast to previous research [37, 40], change in livingalone status was not associated with QoL here. Loneliness at age 79 was, however, associated with scores on the single QoL item at age 90. Numerous studies have supported the view that perceived loneliness is a stronger determinant of subjective wellbeing in older adults than either marital status or living arrangement $[28,72,73]$. Longitudinal data on loneliness and marital status were not available in the LBC1921 sample, preventing investigation of their differential associations with QoL here.

The finding that increased depressive symptoms only significantly contributed to physical QoL, and no other QoL measures, contrasts with our own research [37] and that of others $[36,74]$. One explanation might be that the LBC1921 participants who returned for wave 4 reported relatively few depressive symptoms, with few scoring above the threshold for possible caseness of depression and a median score of 3 out of 21. Current depressive symptoms were significantly associated with all the QoL measures except social QoL, but was excluded from most of the regression analyses due to collinearity. Rerunning the regression analyses using the stepwise entry method 


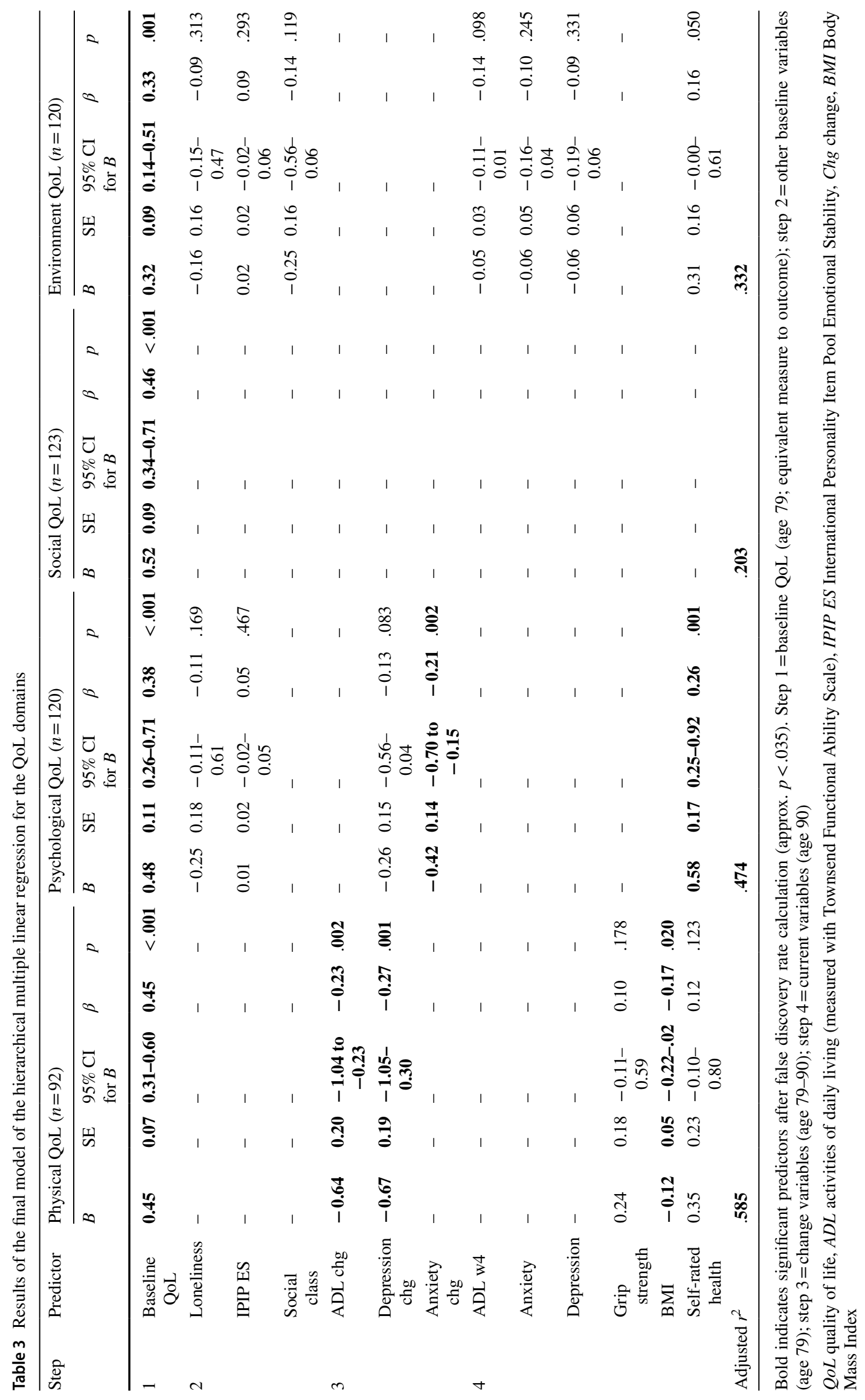


Table 4 Results of the final model of the proportional odds ordinal regression for the QoL and health QoL items

\begin{tabular}{|c|c|c|c|c|c|c|c|c|c|c|}
\hline \multirow[t]{2}{*}{ Predictor } & \multicolumn{5}{|c|}{ QoL item } & \multicolumn{5}{|c|}{ Health QoL item } \\
\hline & Estimate & SE & $p$ & OR & $95 \% \mathrm{CI}$ for OR & Estimate & SE & $p$ & OR & $95 \%$ CI for OR \\
\hline Baseline QoL & 0.32 & 0.48 & .503 & 1.38 & $0.54-3.55$ & 0.88 & 0.26 & .001 & 2.40 & $1.46-3.96$ \\
\hline Loneliness age 79 & -0.91 & 0.31 & .004 & 0.40 & $0.22-0.74$ & - & - & - & - & - \\
\hline ADL change & -0.34 & 0.30 & .246 & 0.71 & $0.40-1.27$ & -0.35 & 0.20 & .074 & 0.70 & $0.48-1.03$ \\
\hline Depression change & -0.32 & 0.29 & .277 & 0.73 & $0.41-1.29$ & 0.14 & 0.21 & .509 & 1.15 & $0.76-1.73$ \\
\hline Lung function age 90 & 0.30 & 0.25 & .230 & 1.35 & $0.83-2.21$ & - & - & - & - & - \\
\hline Self-rated health age 90 & 1.05 & 0.38 & .005 & 2.85 & $1.37-5.94$ & 1.27 & 0.27 & $<.001$ & 3.58 & $2.12-6.03$ \\
\hline
\end{tabular}

Bold indicates significant predictors after false discovery rate calculation (approx. $p<.029$ )

$Q o L$ quality of life, $A D L$ activities of daily living (measured with Townsend Functional Ability Scale), $S E$ standard error

and including current depressive symptoms did not yield different results to those reported here. Nevertheless, minor depressive symptoms do appear to affect QoL. Indeed, Chang et al. [74] suggest that low levels of depressive symptoms are associated with similar demographic and health risks as major depression, and are clinically important as they may indicate subsyndromal depression and other mental health problems. Adverse life events including widowhood, change in financial circumstances, or the onset of disabling illness, are common in old age and can lead to increased depressive symptoms and concomitant decline in QoL. Targeting older adults at these critical points by offering support and/or boosting their internal locus of control might prevent decline in QoL [14, 34]. Health services may also benefit from greater identification of older adults who are experiencing depressive symptoms [33, 34], which may be underreported.

Functional decline, as indicated by increased impairment in ADLs, was strongly associated with physical QoL. Again, this supports previous research [18, 30-32]. Declining functional ability can lead to a loss of independence and selfconfidence, with a consequent decline in mood and QoL. However, some older adults retain high QoL despite low functioning, and they tend to report greater perceived control and use of adaptive coping strategies [75, 76]. Assuming a causal association between perceived control/coping and QoL, services which improve coping abilities and overall functioning in older people-particularly those suffering from chronic illness such as arthritis-might also improve their mood and subsequent QoL $[33,75]$.

Objective health status, as measured by grip strength and lung function, did not significantly contribute to QoL. Selfrated health, however, significantly contributed to psychological QoL, and the two single QoL items. This is unsurprising: previous research suggests self-rated health may be strongly predictive of QoL and other health and wellbeing outcomes in older adults [77-79]. As QoL is largely evaluative, individuals' subjective experiences of their health status may be more influential than objective measures [28].
Stable factors such as social class and personality did not significantly contribute to QoL. However, we have previously shown that these factors predicted age-79 QoL in this group [37]. Given that age-79 QoL was the strongest predictor of age-90 QoL, this suggests that the impact of these stable factors occurs at an earlier timepoint.

The finding that analyses differed by QoL domain supports the notion of QoL as a multi-faceted construct [6, 47]. However, there may be construct overlap between QoL and the predictors described here. Indeed, several WHOQOL-BREF items tap into elements of functional ability and depressive symptoms, asking about ADL, ability to get around, sleep, energy, or ability to concentrate. Nevertheless, the findings highlight the importance of small changes to functional ability, mood, and self-rated health to QoL-all of which are more salient to a carer, health professional, family member, or older person than global QoL.

\section{Strengths and limitations}

The participants were all part of the LBC1921 cohort and living in Scotland. It is possible, therefore, that these results are subject to cohort effects and not generalisable to other countries or time periods [31]. For example, Jivraj and Nazroo [31] found that socioeconomic inequalities and education were stronger predictors of QoL in the USA than in England. However, health-measured by functional ability and chronic conditions-was the strongest predictor of QoL in both countries, supporting the present study's findings. Nevertheless, further replication is essential to enable cohort-based research to influence policymakers or health service provision.

As with all longitudinal studies, the LBC1921 suffered from considerable attrition between waves 1 and 4 . While many non-returning participants had died or were suffering from dementia-related illnesses, a significant number chose not to participate due to poor health or functional limitations [45, 68], as illustrated by the significant differences observed between returners and non-returners on relevant 
baseline measures. Given that functional ability and depression were important predictors of QoL, it is likely that attrition attenuated the study's findings.

The study suffered from a lack of comparative measures between wave 1 and wave 4 . For example, waves $2-4$ of the LBC1921 included measures of loneliness, marital status, living conditions, optimism, resilience, and mental wellbeing. All are potential determinants of QoL, but could not contribute to the present study, which focussed on longitudinal analysis.

The small sample size did not allow for comparative analysis of the results by gender. Although men and women in this sample did not significantly differ on the QoL measures, it is possible that the pattern of associations and predictors may have been different-as noted in our previous research [37]. Future research might, therefore, test for effect modification by gender.

A further limitation - inherent to correlational researchis the strong likelihood of Type I errors. However, FDR control was used to reduce the effect of multiplicity [67].

The study's strengths include its long follow-up and the inclusion of participants who are what is termed as the 'oldest old'. Very few studies have included participants aged 90 or above, and most have fewer participants than this study. Furthermore, the sample's homogeneity eliminates important confounders such as age, and geographical, cultural, and political influences.

\section{Conclusions and implications}

Measures of subjective wellbeing, including QoL, are increasingly popular with policymakers as indicators of population health $[24,80,81]$, and as a key outcome measure of social care and mental health interventions $[82,83]$. The present study suggests that QoL amongst the oldest old is predominantly predicted by QoL measured earlier in the ageing process, indicating that QoL may be relatively stable in old age. This also suggests that $\mathrm{QoL}$ might not be a sensitive measure of short-term change. The results suggest varying patterns of association across different QoL measures, with self-rated health, functional ability, depression, and anxiety, and loneliness all contributing in varying degrees. This study contributes to an expanding body of research suggesting that worsening functional ability, depression, and anxiety, and lower current self-rated health, are strongly predictive of poor QoL in the oldest old. Future research should explore causal pathways and identify factors influencing older adults' perception of their health, the impact of negative life events, and the psychological resources which enhance older adults' ability to cope with the challenges inherent to the ageing process. Interventions to reduce the impact of chronic conditions and illness on subsequent function, and to improve coping and resilience amongst older adults in general, might be beneficial for the health and wellbeing of the oldest old.

Acknowledgements We thank the LBC1921 participants, and the research assistants who contributed to the study. LBC1921 data collection was supported by grants from the Biotechnology and Biological Sciences Research council (BBSRC; Wave 1), and the Chief Scientist Office of the Scottish Government (Wave 4). The work was undertaken in The University of Edinburgh Centre for Cognitive Ageing and Cognitive Epidemiology, which is supported by funding from BBSRC and the Medical Research Council (MRC) as part of the cross council Lifelong Health and Wellbeing Initiative (MR/K026992/1).

Funding Wave 1 of the LBC1921 study received funding support from the Biotechnology and Biological Sciences Research council (15/ SAG09977). Wave 4 of the LBC1921 study received funding support from the Chief Scientist Office of the Scottish Government (ETM/55).

\section{Compliance with ethical standards}

Conflict of interest All authors declare that they have no conflict of interest.

Ethical approval Ethical approval for Wave 1 of the LBC1921 study was obtained from the Lothian Research Ethics Committee (LREC/1998/4/183) and for Wave 4 from the Scotland A Research Ethics Committee (10/S1103/6). All procedures performed in studies involving human participants were in accordance with the ethical standards of the institutional and/or national research committee and with the 1964 Helsinki declaration and its later amendments or comparable ethical standards.

Informed consent Written informed consent was obtained from all individual participants included in the study.

Open Access This article is distributed under the terms of the Creative Commons Attribution 4.0 International License (http://creativeco mmons.org/licenses/by/4.0/), which permits unrestricted use, distribution, and reproduction in any medium, provided you give appropriate credit to the original author(s) and the source, provide a link to the Creative Commons license, and indicate if changes were made.

\section{References}

1. Baltes, P. B., \& Mayer, K. U. (1999). The Berlin Aging Study: Aging from 70 to 100. New York: Cambridge University Press.

2. Wiesmann, U., \& Hannich, H.-J. (2008). A salutogenic view on subjective well-being in active elderly persons. Aging \& Mental Health, 12, 56-65.

3. Depp, C. A., \& Jeste, D. V. (2006). Definitions and predictors of successful aging: A comprehensive review of larger quantitative studies. American Journal of Geriatric Psychiatry, 14, 1.

4. McKee, K. J., \& Schüz, B. (2015). Editorial: Psychosocial factors in healthy ageing. Psychology \& Health, 30, 607-626.

5. WHOQOL Group. (1995). The World Health Organisation Quality of Life assessment (WHOQOL): Position paper from the World Health Organisation. Social Science and Medicine, 41, 1403-1409. 
6. Camfield, L., \& Skevington, S. M. (2008). On subjective wellbeing and quality of life. Journal of Health Psychology, 13(6), 764-775.

7. Diener, E., \& Lucas, R. E. (1999). Personality and subjective wellbeing. In D. Kahneman, E. Diener \& N. Schwarz (Eds.), Wellbeing: The foundations of hedonic personality. New York: Sage.

8. Heller, D., Watson, D., \& Ilies, R. (2004). The role of person versus situation in life satisfaction: A critical examination. Psychological Bulletin, 130, 574-600.

9. Idler, E. L., \& Benyamini, Y. (1997). Self-rated health and mortality: A review of twenty-seven community studies. Journal of Health and Social Behavior, 38, 21-37.

10. Kao, S., Lai, K.-L., Lin, H.-C., Lee, H.-S., \& Wen, H.-C. (2005). WHOQOL-BREF as predictors of mortality: A two-year follow-up study at veteran homes. Quality of Life Research, 14, 1443-1454.

11. Chida, Y., \& Steptoe, A. (2008). Positive psychological well-being and mortality: A quantitative review of prospective observational studies. Psychosomatic Medicine, 70, 741-756.

12. Murray, C., Brett, C. E., Starr, J. M., \& Deary, I. J. (2010). Which aspects of subjectively-reported quality of life are important in predicting mortality beyond known risk factors? The Lothian Birth Cohort 1921 study. Quality of Life Research, 20, 81-90.

13. Steptoe, A., \& Wardle, J. (2012). Enjoying life and living longer: A prospective analysis from the English Longitudinal Study of Ageing. Archives of Internal Medicine, 172, 273-275.

14. Shrestha, S., Stanley, M. A., Wilson, N. L., Cully, J. A., Kunik, M. E., Novy, D. M., et al. (2015). Predictors of change in quality of life in older adults with generalised anxiety disorder. International Psychogeriatrics, 27(7), 1207-1215.

15. Schilling, O. (2006). Development of life satisfaction in old age: Another view on the "Paradox". Social Indicators Research, 75, 241-271.

16. Mollenkopf, H., \& Walker, A. (Eds.). (2007). Quality of life in old age: International and multidisciplinary perspectives. Dordrecht: Springer.

17. Mroczek, D. J., \& Spiro, A. (2005). Change in life satisfaction during adulthood: Findings from the Veteran Affairs Normative Aging Study. Journal of Personality and Social Psychology, 88, 189-202.

18. Netuveli, G., Wiggins, R. D., Hildon, Z., Montgomery, S. M., \& Blane, D. (2006). Quality of life at older ages: Evidence from the English longitudinal study of aging (wave 1). Journal of Epidemiology and Community Health, 60, 357-363.

19. Blanchflower, D. G., \& Oswald, A. J. (2008). Is well-being U-shaped over the life cycle? Social Science and Medicine., 66, 1733-1749.

20. Stone, A. A., Schwartz, J. E., Broderick, J. E., \& Deaton, A. (2010). A snapshot of the age distribution of psychological wellbeing in the United States. Proceedings of the National Academy of Sciences of the United States of America, 107, 9985-9990.

21. Baird, B. M., Lucas, R. E., \& Donnellan, M. B. (2010). Life satisfaction across the lifespan: Findings from two nationally representative panel studies. Social Indicators Research, 99, 183-203.

22. Jivraj, S., Nazroo, J., Vanhoutte, B., \& Chandola, T. (2014). Aging and subjective well-being in later life. Journals of Gerontology, Series B: Psychological Sciences and Social Sciences, 69(6), 930-941.

23. Eid, M., \& Diener, E. (2004). Global judgements of subjective well-being: Situational variability and long-term stability. Social Indicators Research, 65, 245-277.

24. Kahneman, D., \& Krueger, A. B. (2006). Developments in the measurement of subjective well-being. Journal of Economic Perspectives, 20, 3-24.

25. Clark, A. E., Diener, E., Georgellis, Y., \& Lucas, R. E. (2008). Lags and leads in life satisfaction: A test of the baseline hypothesis. Economic Journal, 118, F222-F243.
26. Diener, E., Suh, E. M., Lucas, R. E., \& Smith, H. L. (1999). Subjective well-being: Three decades of progress. Psychological Bulletin, 125, 276-302.

27. Schimmack, U., Diener, E., \& Oishi, S. (2002). Life-satisfaction is a momentary judgment and a stable personality characteristic: The use of chronically accessible and stable sources. Journal of Personality, 70, 345-384.

28. Webb, E., Blane, D., McMunn, A., \& Netuveli, G. (2011). Proximal predictors of change in quality of life at older ages. Journal of Epidemiology and Community Health, 65, 542-547.

29. Clare, L., Woods, R. T., Nelis, S. M., Martyr, A., Marková, I. S., Roth, I., et al. (2014). Trajectories of quality of life in early-stage dementia: Individual variations and predictors of change. International Journal of Geriatric Psychiatry, 29, 616-623.

30. Bowling, A., Banister, D., Sutton, S., Evans, O., \& Windsor, J. (2002). A multidimensional model of the quality of life in older age. Aging \& Mental Health, 6(4), 355-371.

31. Jivraj, S., \& Nazroo, J. (2014). Determinants of socioeconomic inequalities in subjective well-being in later life: A cross-country comparison in England and the USA. Quality of Life Research, 23, 2545-2558.

32. Davis, J. C., Bryan, S., Best, J. R., Li, L. C., Hsu, C. L., Gomez, C., et al. (2015). Mobility predicts change in older adults' healthrelated quality of life: Evidence from a Vancouver falls prevention prospective cohort study. Health and Quality of Life Outcomes, $13,101$.

33. Chan, S. W., Chiu, H. F. K., Chien, W., Goggins, W., Thompson, D., \& Hong, B. (2009). Predictors of change in health-related quality of life among older people with depression: A longitudinal study. International Psychogeriatrics, 21(6), 1171-1179.

34. Grundy, E., \& Bowling, A. (1999). Enhancing the quality of extended life years. Identification of the oldest old with a very good and very poor quality of life. Aging \& Mental Health, 3(3), 199-212.

35. Bain, G. H., Lemmon, H., Teunisse, S., Starr, J. M., Fox, H. C., Deary, I. J., et al. (2003). Quality of life in healthy old age: Relationships with childhood IQ, minor psychological symptoms and optimism. Social Psychiatry and Psychiatric Epidemiology, 38, 632-636.

36. Lapid, M. I., Rummans, T. A., Boeve, B. F., McCormick, J. K., Pankratz, V. S., Cha, R. H., et al. (2011). What is the quality of life of the oldest old? International Psychogeriatrics, 23(6), 1003-1010.

37. Brett, C. E., Gow, A. J., Corley, J., Pattie, A., Starr, J. M., \& Deary, I. J. (2012). Psychosocial factors and health as determinants of quality of life in community-dwelling older adults. Quality of Life Research, 21, 505-516.

38. Pinquart, M., \& Sörensen, S. (2000). Influences of socioeconomic status, social network, and competence on subjective well-being in later life: A meta-analysis. Psychology and Aging, 15, 187-224.

39. Seymour, D. G., Starr, J. M., Fox, H. C., Lemmon, H. A., Deary, I. J., Prescott, G. J., et al. (2008). Quality of life and its correlates in octogenarians. Use of the SEIQoL-DW in Wave 5 of the Aberdeen Birth Cohort 1921 Study (ABC1921). Quality of Life Research, 17, 11-20.

40. Blane, D., Higgs, P., Hyde, M., \& Wiggins, R. D. (2004). Life course influences on quality of life in early old age. Social Science \& Medicine, 58, 2171-2179.

41. Smith, J., \& Baltes, P. B. (1997). Profiles of psychological functioning in the old and oldest old. Psychology and Aging, 12, 458-472.

42. Age, U. K. (2013). Improving later life: Understanding the oldest old. London: Age UK.

43. Deary, I. J., Gow, A. J., Pattie, A., \& Starr, J. M. (2012). Cohort profile: The Lothian Birth Cohorts of 1921 and 1936. International Journal of Epidemiology, 41(6), 1576-1584. 
44. Scottish Council for Research in Education. (1933). The intelligence of Scottish children: A national survey of an age-group. London: University of London Press.

45. Taylor, A. M., Pattie, A., \& Deary, I. J. (2018). Cohort profile update: The Lothian Birth Cohorts of 1921 and 1936. International Journal of Epidemiology, 1-19.

46. Sibbett, R. A., Russ, T. C., Pattie, A., Starr, J. M., \& Deary, I. J. (2018). Does incipient dementia explain normal cognitive decline determinants? Lothian Birth Cohort 1921. Psychology and Aging, $33,674-684$.

47. WHOQOL Group. (1994). Development of the WHOQOL: Rationale and current status. International Journal of Mental Health, 23(3), 24-56.

48. World Health Organization. (1997). WHOQOL-BREF. Introduction, administration, scoring, and generic version of the assessment. MNH/PSF 97.4 WHO, Geneva.

49. WHOQOL Group. (1998). Development of the World Health Organisation WHOQOL-BREF quality of life assessment. Psychological Medicine, 28(3), 551-558.

50. Skevington, S. M., Lotfy, M., \& O'Connell, K. A. (2004). The World Health Organization's WHOQOL-BREF quality of life assessment: Psychometric properties and results of the international field trial. A Report from the WHOQOL Group. Quality of Life Research, 13, 299-310.

51. Zigmond, A. S., \& Snaith, R. P. (1983). The Hospital Anxiety and Depression Scale. Acta Psychiatrica Scandinavia, 67, 361-370.

52. Bjelland, I., Dahl, A. A., Haug, T. T., \& Neckelmann, D. (2002). The validity of the Hospital Anxiety and Depression Scale: An updated literature review. Journal of Psychosomatic Research, 52, 69-77.

53. Townsend, P. (1979). Poverty in the United Kingdom: A survey of household resources and standards of living. Harmondsworth: Penguin.

54. Sprott, R. L. (2010). Biomarkers of aging and disease: Introduction and definitions. Experimental Gerontology, 45, 2-4.

55. Lara, J., Cooper, R., Nissan, J., Ginty, A. T., Khaw, K.-T., Deary, I. J., et al. (2015). A proposed panel of biomarkers of healthy ageing. BMC Medicine, 13, 222.

56. Brett, C. E., \& Deary, I. J. (2015). How to... obtain accurate objective measurements of health at a distance. Health Psychology Update.

57. Bohannon, R. W. (2008). Hand-grip dynamometry predicts future outcomes in ageing adults. Journal of Geriatric Physical Therapy, $31,3-10$

58. Deary, I. J., Johnson, W., Gow, A. J., Pattie, A., Brett, C. E., Bates, T. C., et al. (2011). Losing one's grip: A bivariate growth curve model of grip strength and nonverbal reasoning from age 79 to 87 years in the Lothian Birth Cohort 1921. The Journals of Gerontology, Series B: Psychological Sciences and Social Sciences, 66(6), 699-707.

59. Roberts, M. H., \& Mapel, D. W. (2012). Limited lung function: Impact of reduced peak expiratory flow on health status, healthcare utilisation, and expected survival in older adults. American Journal of Epidemiology, 176(2), 128-134.

60. Knudtson, M. D., Klein, B. E. K., \& Klein, R. (2009). Biomarkers of aging and falling: The Beaver Dam eye study. Archives of Gerontology and Geriatrics, 49(1), 22-26.

61. Yan, L. L., Daviglus, M. L., Liu, K., Pirzada, A., Garside, D. B., Schiffer, L., et al. (2004). BMI and health-related quality of life in adults 65 years and older. Obesity Research, 12, 69-76.

62. General Register Office. (1956). Census 1951: Classification of occupations. London: HMSO.

63. Goldberg, L. R. (1992). The development of markers for the BigFive factor structure. Psychological Assessment, 4, 26-42.

64. Goldberg, L. R. (2001). International Personality Item Pool. http://ipip.ori.org/. Accessed 5 June 2018.

65. Gow, A. J., Whiteman, M. C., Pattie, A., \& Deary, I. J. (2005). Goldberg's IPIP Big-Five factor markers: Internal consistency and concurrent validation in Scotland. Personality and Individual Differences, 39, 317-329.

66. Steel, P., Schmidt, J., \& Shultz, J. (2008). Refining the relationship between personality and subjective well-being. Psychological Bulletin, 134, 138-161.

67. Benjamini, Y., \& Hochberg, Y. (1995). Controlling the false discovery rate: A practical and powerful approach to multiple testing. Journal of the Royal Statistical Society Series B (Statistical Methodology), 57, 289-300.

68. Deary, I. J., Pattie, A., \& Starr, J. M. (2013). The stability of intelligence from age 11 to age 90 years: The Lothian Birth Cohort of 1921. Psychological Science, 24, 2361-2368.

69. Veenhoven, R. (2008). Healthy happiness: Effects of happiness on physical health and the consequences for preventive health care. Journal of Happiness Studies, 9, 449-469.

70. Oswald, A. J., \& Powdthavee, N. (2008). Does happiness adapt? A longitudinal study of disability with implications for economists and judges. Journal of Public Economics, 92, 1061-1077.

71. Cohen, L., Wimbush, E., Myers, F., Macdonald, W., \& Frost, H. (2014). Optimising older people's quality of life: An outcomes framework. Strategic Outcomes Model. Edinburgh: NHS Health Scotland.

72. Golden, J., Conroy, R. M., Bruce, I., Denihan, A., Greene, E., Kirby, M., et al. (2009). Loneliness, social support networks, mood and well-being in community-dwelling elderly. International Journal of Geriatric Psychiatry, 24, 694-700.

73. Gow, A. J., Pattie, A., Whiteman, M. C., Whalley, L. J., \& Deary, I. J. (2007). Social support and successful aging: Investigating the relationship between lifetime cognitive change and life satisfaction. Journal of Individual Differences, 28, 103-115.

74. Chang, Y.-C., Ouyang, W.-C., Lu, M.-C., Wang, J.-D., \& Hu, S. C. (2016). Levels of depressive symptoms may modify the relationship between the WHOQOL-BREF and its determining factors in community-dwelling older adults. International Psychogeriatrics, 28(4), 591-601.

75. Bowling, A., Seetai, S., Morris, R., \& Ebrahim, S. (2007). Quality of life among older people with poor functioning. The influence of perceived control over life. Age and Ageing, 36, 310-315.

76. Carpentieri, J. D., Elliott, J., Brett, C. E., \& Deary, I. J. (2016). Adapting to ageing: A mixed methods study of the role of Selection, Optimisation and Compensation in the maintenance of high wellbeing. Journal of Gerontology, Series B: Psychological Sciences and Social Sciences, 72, 351-361.

77. Subramanian, S. V., Kim, D., \& Kawachi, I. (2005). Covariation in the socioeconomic determinants of self rated health and happiness: A multivariate multilevel analysis of individuals and communities in the USA. Journal of Epidemiology and Community Health, 59(8), 664-669.

78. Borim, F. S. A., Neri, A. L., Francisco, P. M. S. B., \& de Barros, M. B. A (2014). Dimensions of self-rated health in older adults. Revista de Saude Publica, 48(5), 714-722.

79. DeSalvo, K. B., Bloser, N., Reynolds, K., He, J., \& Munter, P. (2006). Mortality prediction with a single general self-rated health question: A meta-analysis. Journal of General Internal Medicine, 21(3), 267-275.

80. Helliwell, J. F., Layard, R., \& Sachs, J. (Eds.). (2015). World happiness report 2015. New York: Sustainable Development Solutions Network.

81. Powdthavee, N. (2015). Would you like to know what makes people happy? An overview of the data sets on subjective well-being. Australian Economic Review, 48(3), 314-320.

82. IsHak, W. W., Greenberg, J. M., Balayan, K., Kapitanski, N., Jeffrey, J., Fathy, H., et al. (2011). Quality of life: The ultimate outcome measure of interventions in major depressive disorder. Harvard Reviews Psychiatry, 19(5), 229-239. 
83. Bowling, A. (2014). Quality of life: Measures and meanings in social care research. London: NIHR School for Social Care Research.
Publisher's Note Springer Nature remains neutral with regard to jurisdictional claims in published maps and institutional affiliations. 